\title{
Length scales in solutions of the Navier-Stokes equations
}

\author{
M V Bartuccelli†§, C R Doeringt, J D Gibbon† and S J A Malham $\dagger$ \\ $\dagger$ Department of Mathematics, Imperial College, London SW7 2BZ, UK \\ $\ddagger$ Department of Physics and Clarkson Institute for Statistical Physics, Clarkson University, \\ Potsdam, NY 13699-5820, USA.
}

Received 23 November 1992

Recommended by P Constantin

\begin{abstract}
A set of ladder inequalities for the $2 d$ and $3 d$ forced Navier-Stokes equations on a periodic domain $[0, L]^{d}$ is developed, leading to a natural definition of a set of length scales. We discuss what happens to these scales if intermittent fluctuations in the vorticity field occur, and we consider how these scales compare to those derived from the attractor dimension and the number of determining modes. Our methods are based on estimates of ratios of norms which appear to play a natural role and which make many of the calculations comparatively easy. In $3 d$ we cannot preclude length scales which are significantly shorter than the Kolmogorov length. In $2 d$ our estimate for a length scale $\ell$ turns out to be
\end{abstract}

$$
(\ell / L)^{-2} \leqslant c \mathcal{G}(1+\log \mathcal{G})^{1 / 2}
$$

where $\mathcal{G}$ is the Grashof number. This estimate of $\ell$ is shorter than that derived from the attractor dimension. The reason for this is discussed in detail.

PACS numbers: 4610

\section{Introduction}

The problem addressed in this paper concerns length scales arising in turbulence. To make both mathematical and physical sense, these scales should arise naturally out of the Navier-Stokes equations and should also make a connection with the length scales used. in conventional theories of turbulence. To be natural scales in a flow, they should also take into account, as much as possible, fluctuations in the vorticity away from spatial and temporal averages. Intermittent events may be rare, but may drive structures to substantially smaller scales than those associated with the long time periods when the flow remains close to these averages. Ideally, estimates for these scales will also be associated with results for the $3 d$ Euler equations concerning the possible breakdown of regularity. In their paper on the breakdown of smooth solutions of the $3 d$ incompressible Euler equations, Beale et al [1] have shown that $\int_{0}^{t}\|D u\|_{\infty}(\tau) \mathrm{d} \tau$ (converted to $\left.\int_{0}^{t}\|\omega\|_{\infty}(\tau) \mathrm{d} \tau\right)$ controls the possible breakdown of regularity in the $3 d$ Euler equations. It has long been thought that a loss of regularity in the $3 d$ Euler equations could have a major influence on the onset of NavierStokes turbulence (see the reviews by Majda [2,3]).

Whether singularities actually form in the $3 d$ Euler equations is still an open question, but Beale, Kato and Majda's identification of $\int_{0}^{t}\|D u\|_{\infty}(\tau) \mathrm{d} \tau$ (or $\int_{0}^{t}\|\omega\|_{\infty}(\tau) \mathrm{d} \tau$ ) as the 
controlling object raises several questions about the role of $\|D u\|_{\infty}$ in the occurrence of vorticity fluctuations in the $3 d$ Navier-Stokes equations. Even if the flow remains regular (which is an assumption in itself), intermittency may cause large fluctuations in the flow, in which $\|D u\|_{\infty}$ deviates a significant distance away from its spatial average. This opens the question of whether scales much deeper than the Kolmogorov length may actually be achieved.

The aim of this paper is fivefold:

- To state and prove a so-called ladder theorem which takes a proper and accurate account of the force driving the flow.

- From this ladder theorem to define a length scale which is given naturally by the Navier-Stokes equations themselves and which can be logically connected to more familiar definitions of dissipation lengths in turbulence theory.

- To compare the estimates derived from this definition of a length scale with that computed from other definitions such as the scale found from the attractor dimension [4-6], the number of determining modes (see [7-9]), the scale found in [10] and that found in $[11,12]$.

- To see if the Kolmogorov length $\lambda_{\mathrm{K}}=\left(v^{3} / \varepsilon\right)^{1 / 4}$ has any meaning in this picture and to see if rare intermittent fluctuations in the vorticity drive structures to length scales smaller than $\lambda_{\mathrm{K}} . \varepsilon$. in the definition of $\lambda_{\mathrm{K}}$ is taken to be the space-time average of the energy dissipation rate.

- To see how well this definition of a 'natural scale' picks up or loses information on rare fluctuations through the taking of time averages.

This paper is an attempt to address these questions in as rigorous yet simple a manner as possible. For the reader who is not interested in technical questions, the proofs of the various theorems are left to section 5 . Our study begins with the Navier-Stokes equations, with zero momentum $\int_{\Omega} u \mathrm{~d}^{d} x=0$, on a periodic domain $\Omega \equiv[0, L]^{d}$, where $d$ is the spatial dimension

$$
\begin{aligned}
& u_{t}+u \cdot \nabla u=v \Delta u-\nabla p+f \\
& \operatorname{div} u=0 .
\end{aligned}
$$

The divergence free periodic forcing function $f(x)$ is independent of $t$ with a cut-off in its wavenumber spectrum at $k_{\max }=2 \pi / \lambda_{f}$. Hence $\lambda_{f}$ is the smallest scale in the forcing. To understand how solutions behave as $t$ increases, it is necessary to study the seminorms

$$
H_{N}=\sum_{i=1}^{d}\left\|D^{N} u_{i}\right\|_{2}^{2}
$$

where $D^{N}$ stands for the sum of all derivatives in $d$ dimensions of order $N$ and $\|g\|_{2}^{2}=$ $\int_{\Omega}|g|^{2} \mathrm{~d} x$ is the $L^{2}$-norm. To include the forcing in a rational way, we introduce the natural time scale $\tau_{0}=L^{2} / v$ and define velocity and vorticity fields associated with the forcing $u_{f}=\tau_{0} f$ and $\omega_{f}=\operatorname{curl} u_{f}$. The combination

$$
F_{N}=H_{N}+\sum_{i=1}^{d}\left\|D^{N} u_{f, i}\right\|_{2}^{2}
$$

therefore includes the forcingt and its derivatives. The $F_{N}$ satisfy a simple differential 'ladder inequality' which is demonstrated in

$\dagger$ Recall that there is a cut-off in the wavenumber spectrum of the forcing at $k_{\max }=2 \pi / \lambda_{f}$. 
Theorem 1. For $N \geqslant 1,1 \leqslant s \leqslant N$ and $d=2,3$

$$
\frac{1}{2} \dot{F}_{N} \leqslant-v \frac{F_{N}^{1+1 / s}}{F_{N-s}^{1 / s}}+\left(c_{N, s}\|D u\|_{\infty}+v \lambda_{0}^{-2}\right) F_{N}
$$

where

$$
\lambda_{0}^{-2}=L^{-2}+\lambda_{f}^{-2}
$$

and $c_{N, s}$ are constants which depend only on $N, s$ and $d$.

This theorem $\ddagger$ is a generalization of the ladder theorem $\S$ in [10]. Here we have a more precise statement of the effect of the forcing with the domain length and the cutoff scale in the forcing appearing in $\lambda_{0}$. To try to get $F_{0}$ as the 'bottom rung' of either of our ladders, it is necessary to take $s=N$. For either dimension $d=2$ or $d=3$, the negative definite terms are never quite strong enough to control the positive definite terms. Therefore, we are unable to prove the existence of an absorbing set in the appropriate function space solely with $s=N$. There are several different ways of trying to get around this, some of which are sharper than others, but all fail with $s=N$. Even for the $2 d$ case one is forced, as in $3 d$, to take $s=N-1$. In both the $2 d$ and $3 d$ cases therefore, $F_{\mathbf{1}}$ is the 'bottom rung' of the ladder. In $2 d$, this quantity can be bounded above a priori (a result which is well known) thereby establishing regularity for the $2 d$ case. section 4.2 deals with the $2 d$ case further. Unfortunately, in $3 d$ no a priori bound is known, in general, for the $H_{1}$ norm or any higher norms.

\subsection{A natural definition of length scales}

To see how theorem 1 naturally gives a set of length scales through the $F_{N}$, we firstly look at the problem through a Fourier series expansion. Then we show that the ladder (1.5) gives a natural length scale associated with moments of the power spectrum.

(a) Fourier modes: Take a function $g$ in $3 d$ and write it in terms of its Fourier series

$$
g=\sum_{k=1}^{\infty} a_{k} \exp (\mathrm{i} k x)=\left(\sum_{k<k}+\sum_{k>\kappa}\right) a_{k} \exp (\mathrm{i} k \cdot x)
$$

where the wavenumber $\kappa$, as yet undetermined, divides between 'low' modes $k<\kappa$ and 'high' modes $k>\kappa$. After an application of the Schwarz inequality, this can be written as

$$
|g| \leqslant\left(\sum_{k<\kappa} 1^{2}\right)^{1 / 2}\left(\sum_{k<\kappa}\left|a_{k}\right|^{2}\right)^{1 / 2}+\left(\sum_{k>\kappa} k^{-2 s}\right)^{1 / 2}\left(\sum_{k>\kappa}\left|k^{s} a_{k}\right|^{2}\right)^{1 / 2}
$$

for some $s>3 / 2$. This can be written as

$$
\|g\|_{\infty} \leqslant c\left(\kappa^{3 / 2}\|g\|_{2}+\kappa^{3 / 2-s}\left\|D^{s} g\right\|_{2}\right)
$$

Now we take $g=D^{r} u$ and $s+r=N$ and use (1.4) to get

$$
\left\|D^{r} u\right\|_{\infty} \leqslant c\left(\kappa^{3 / 2} F_{N-s}^{1 / 2}+\kappa^{3 / 2-s} F_{N}^{1 / 2}\right)
$$

† See section 5 for the proof.

$\S$ There is also an equivalent theorem (see [10]) with $v^{-1}\left\langle\|u\|_{\infty}^{2}\right\rangle$ instead of $\|D u\|_{\infty}$ and $-v / 2$ instead of $-v$. 
whence equal influence between the low and high modes determines $\kappa$ to be (with labels $N$ and $r=N-s$ )

$$
\kappa_{N, r}^{2 s}=\frac{F_{N}}{F_{r}} .
$$

With this choice of $k$ in (1.10), we recover a well known calculus inequality.

(b) Moments of the power spectrum: A scale in turbulence theory may be determined in the following way: through the energy in the flow (equivalent to $H_{0}$ ),

$$
E(t)=\int_{\Omega}|u(x, t)|^{2} \mathrm{~d} x
$$

we can use Parseval's theorem

$$
E(t)=\int|\hat{u}(k, t)|^{2} \mathrm{~d} k \equiv \int E(k, t) \mathrm{d} k
$$

to get the instantaneous energy spectrum $E(k, t)$. This defines the distribution of energy among length scales and also allows us to consider the normalized 'probability distribution'

$$
P(k, t)=\frac{E(k, t)}{E(t)}
$$

Time dependent moments of this distribution can then be written as

$$
\left\langle k^{2 N}\right\rangle_{\mathrm{s.a} .}=\int|k|^{2 N}|\hat{u}(k, t)|^{2} d k / \int|\hat{u}(k, t)|^{2} \mathrm{~d} k=H_{N} / H_{0}
$$

where the s.a. on $\left\langle k^{2 N}\right\rangle_{\text {s.a. }}$ stands for 'spatial average'. The relevant time dependent wavelength scale $\lambda_{N}$ associated with this quantity is

$$
\lambda_{N}^{-1} \sim\left[\left\langle k^{2 N}\right\rangle_{\text {s.a.t }}\right]^{1 / 2 N} .
$$

Not only can the forcing be included in the definitions to make the $H_{N}$ 's into $F_{N}$ 's, but the ratio of moments

$$
\frac{\left\langle k^{2 N}\right\rangle_{\text {s.a. }}}{\left\langle k^{2 r}\right\rangle_{\text {s.a. }}}=\frac{F_{N}}{F_{r}}
$$

also naturally defines a length scale

$$
\text { (length })^{-1} \sim\left(\frac{F_{N}}{F_{r}}\right)^{1 / 2(N-r)}
$$

which is the same as that defined in (1.11).

Clearly, we can define a set of 'wavenumbers' defined by the ratio of moments above. 
Definition 1.

$$
\kappa_{N, r}(t)=\left(\frac{F_{N}}{F_{r}}\right)^{1 / 2(N-r)} \cdot r=N-s \geqslant 0 .
$$

Identifying $r=N-s$, it is noteworthy that if one divides equation (1.5) in theorem 1 through by $F_{N}$, then the square of our time dependent 'wavenumbers' appear in the Laplacian term. It is convenient and natural, therefore, to operate the time average $\langle\cdot\rangle$, defined by

$$
\langle g\rangle=\sup _{g(0)} \lim _{t \rightarrow \infty} \frac{1}{t} \int_{0}^{t} g(\tau) \mathrm{d} \tau
$$

on the square of these objects to get an associated set of inverse squared lengths.

\section{Definition 2.}

$$
\ell_{N, r}^{-2} \equiv\left\langle\kappa_{N, r}^{2}\right\rangle=\left\langle\left(\frac{F_{N}}{F_{r}}\right)^{1 /(N-r)}\right\rangle
$$

The precise statement is

Theorem 2. Suppose the velocity field $u_{f}=\tau_{0} f$ associated with the forcing $f(x)$ satisfies $\sum_{i=1}^{d}\left\|D^{N} u_{f, i}\right\|_{2}^{2}>1$ for each value of $N$. Then for $d=3$ and $N \geqslant 3$

$$
\ell_{N, r}^{-2} \equiv\left\langle\kappa_{\uparrow, r}^{2}\right\rangle \leqslant c_{N, r} \nu^{-1}\left\langle\|D u\|_{\infty}\right\rangle+\lambda_{0}^{-2}
$$

Furthermore $\dagger$, for $N=2$ and $N=1$,

$$
\ell_{2,1}^{-2} \equiv\left\langle\kappa_{2,1}^{2}\right\rangle \leqslant c_{2,1} v^{-1}\left\langle\|\omega\|_{\infty}\right\rangle+\lambda_{0}^{-2}
$$

and

$$
\ell_{1,0}^{-2} \equiv\left\langle\kappa_{1,0}^{2}\right\rangle \leqslant \lambda_{0}^{-2}
$$

Note firstly that there is only a minor restriction on the forcing function and secondly these results depend on $d$ only in the constants. The proof of this theorem is given in section 5. In fact for $N=1$ this is just a restatement of Leray's inequality. For $N \geqslant 3$, the upper bound on $\ell_{N, r}^{-2}$ is uniform in $(N, r)$, except in the constant, so we can consider all these scales to be essentially the same; namely.

$$
\ell \sim \sqrt{\frac{v}{\left(\|D u\|_{\infty}\right)}}
$$

with a correction term in $\lambda_{0}^{-2}$. While (1.25) represents an estimate for the scale in the fluid, the terms in $\lambda_{0}^{-2}$ also contain spectral information on the forcing.

$\dagger$ The results are the same in $2 d$ except for the case $(N, r) \equiv(2,1)$. In that case $c_{2,1}=0$ whence $\left\langle\kappa_{2,1}^{2}\right\} \leqslant \lambda_{0}^{-2}$. The $\|\omega\|_{\infty}$ term is missing because the $\omega . \nabla$ term is zero in $2 d$. 


\subsection{A comparison of different scales}

There are a variety of different ways of computing a natural scale in a Navier-Stokes flow. Historically, length scales have been identified within the wavenumber spectrum. More recently the attractor dimension $[13,5,6]$ and the number of determining modes, which was a concept first introduced by Foias and Prodi [14] (see also [7-9]) have been interpreted in terms of length scales in the flow. Each measures different properties of the dynamics on a universal attractor, if the latter exists. In the $2 d$ Navier-Stokes case this attractor exists and much effort has gone into understanding its properties [4-6]. No proof for an attractor exists in the $3 d$ case because of the inability to control the $H_{1}$ norm. Consequently, one is left with estimates for length scales, calculated in terms of quantities such as $\left\langle\|D u\|_{\infty}\right\rangle$, which have no known a priori upper bound in terms of externally controlled quantities such as the Reynolds, Rayleigh or Grashof numbers. The history of the Navier-Stokes equations since the early 1960 s has been an elusive search for tegularity [15-18,5]. It is well known that it can only be proved that a flow will remain regular for a finite time; thereafter the upper bounds diverge and any information, other than that about weak solutions, is lost. Consequently, predictions about the flow and its natural scales are based on the assumption that various norms remain bounded for sufficiently large times for time averages to make sense. This assumption of regularity cannot be justified solely on mathematical grounds alone but, in physical terms, it is nevertheless used to predict properties of $3 d$ turbulence which cannot be found by other methods [19]. This paper is written in that spirit.

The length scale given in (1.25), which comes out in terms of $v^{-1}\left\langle\|D u\|_{\infty}\right\}$, is an intensive quantity as it is formally independent of the system volume. That is, this quantity is the same for a number of identical independent systems taken together, in contrast with 'extensive' quantities like the total kinetic energy $\left(L^{2}\right.$ squared) which are additive. It coincides with the $3 d$ Navier-Stokes scale calculated by Constantin, Foias and Temam using the attractor dimension $[4,13]$ (see also their respective books [5] and [6]). It also coincides with the scale calculated in [10], derived without taking the forcing into account, and also with the scale found by Henshaw, Kreiss and Reyna $[11,12]$ using different methods. We discuss these comparisons in more detail in the conclusion in section 4.

As we will also see in that section, the $2 d$ case gives more explicit results as regularity is already proven. Our final result for $\left\langle\kappa_{N, r}^{2}\right)$ in $2 d$ with $N \geqslant 3$ is (see theorem 7 section 4)

$$
\left(\frac{\ell_{N, r}}{L}\right)^{-2} \leqslant c \mathcal{G}(1+\dot{\log } \mathcal{G})^{1 / 2}
$$

where $\mathcal{G}$ is the Grashof number. This scales coincide with the length scale defined by the number of determining modes given in [7,8].

Our main concern here has been the definition of a natural scale which has been made through the taking of a time average. While this scale might be natural in the way it appears from the Navier-Stokes equations, nevertheless it is not necessarily the minimum scale. The fact that we have taken an average means that some information must have been lost and therefore it is not clear that the deepest fluctuations caused by intermittent events have been resolved. To resolve these it is necessary to look at the $\kappa_{N, r}(t)$ themselves without the operation of the time average. Estimates for $\overline{\lim }_{t \rightarrow \infty} \kappa_{N, r}(t)$ are now relevant in this case. In fact, in the $2 d$ case, we find that

$$
\overline{\lim }_{t \rightarrow \infty} \kappa_{N, r}(t) \leqslant c L^{-1} \mathcal{G}^{2}
$$

showing that there may' be a scale smaller than $\mathcal{G}^{-1 / 2}(1+\log \mathcal{G})^{-1 / 4}$. This matter is also addressed further in section 4 where the known regularity of the $2 d$ Navier-Stokes equations 
makes matters easier to interpret. A further example used in that section is that of the complex Ginzburg Landau equation where we illustrate the contrast between estimates for $\left\langle\kappa_{N, r}^{2}\right\rangle$ and $\varlimsup_{t \rightarrow \infty} \mathcal{K}_{N, r}(t)$.

For the moment, while this problem and a comparison with the other measures of dimension is left to section 4 , it is instructive to see how our definition of a length scale compares with the way time averages are taken of moments to define a length scale in turbulence theory. In that approach, it is usual to consider the time averaged energy spectrum $\langle E(k)\rangle$ (which is the quantity which is supposed to decay algebraically in an inertial range). The relevant length scales are defined in terms of the average distribution of energy

$$
\langle P\rangle(k)=\frac{\left\langle E\left(k_{,}\right)\right\rangle}{\int \mathrm{d} k^{\prime}\left\langle E\left(k^{\prime}, .\right)\right\rangle} .
$$

This, of course, is not the same as time averaged instantaneous distribution of energy

$$
\langle P(k, .)\rangle=\left\langle\frac{E(k, .)}{\int \mathrm{d} k^{\prime} E\left(k^{\prime}, .\right)}\right\rangle .
$$

A conventional definition of a length scale is calculated via $\langle P\rangle(k)$ and so a ratio of time averages of the $F_{N}$ and $F_{r}$ would occur. In our natural (for Navier-Stokes) but slightly unconventional scale defined in (1.21) we have a time average of a ratio. Moments computed by taking the time average last are going to be more sensitive to rare, deep fluctuations in the vorticity down to short scales than those computed from the time-averaged energy spectrum. This is because, all other things being equal, rare events contribute little to the average energy spectrum. Moreover, when a significant fraction of energy is at high wavenumbers, the (relative) energy dissipation is necessarily higher, so these events will typically be characterized by lower than normal total energy. They will then count little toward the time average of the energy spectrum. Dividing by the total energy before averaging therefore amplifies the role of the low energy but high wavenumber configurations in the distribution, skewing the distribution towards high wavenumbers.

Consequently, despite our slightly unconventional definition, we assert that the scales defined by computing $\left\langle\kappa_{N, r}^{2}\right\rangle$ are natural to the Navier-Stokes equations and are likely to be more relevant to intermittent fluctuations than scales determined by the time-averaged energy spectrum (for example, the Kolmogorov length). As we have pointed out in the paragraphs above, however, whether this sensitivity goes far enough to pick up information on the smallest length scale is a different matter and is a point left for discussion in section 4.

\section{Length scales during intermittency}

As we have already discussed in section 1.2, predictions about the flow and its natural scales are based on the assumption that various norms remain bounded for sufficiently large times for time averages to make sense. We are particularly interested in the possibility that even if the flow remains regular (an assumption in itself), rare, large intermittent fluctuations or bursts in the vorticity field away from averages may nevertheless occur. If this is the case, a flow may remain close to its spatial average for large periods of time but, in shott intervals, bursts in the vorticity field might cause the deep excursions mentioned above. The deeper they are, the rarer these events must be to avoid violating averages. This type of intermittency in the flow would likely be unpredictable. Because of the relatively long quiescent periods between these events, one has to think of how to compute a length scale 
associated with the flow during these periods and then a second shorter scale associated with a burst if or when it occurs. It is in this situation that we would like to investigate the role of the Kolmogorov length $\lambda_{\mathrm{K}}$. In particular, we would like to see whether $\lambda_{\mathrm{K}}$ is a natural scale, as is commonly assumed, or whether significantly shorter scales than this could possibly occur.

In the estimate from theorem $2, v^{-1}\left\langle\|D u\|_{\infty}\right\rangle$ is distinguished by the fact that it is expected to be an intensive quantity; that is, independent of system size. Formally, Constantin, Foias and Temam [4-6] estimate a Kolmogorov length from this quantity by defining an energy dissipation rate $\varepsilon_{\infty}=v\left(\|D u\|_{\infty}\right\}^{2}$. Substitution of $\varepsilon_{\infty}$ into theorem 2 gives

$$
\ell_{N, r}^{-2} \leqslant c_{N, r} \lambda_{\mathrm{K}, \infty}^{-2}+\lambda_{0}^{-2}
$$

with

$$
\lambda_{\mathrm{K}, \infty}=\left(\frac{\nu^{3}}{\varepsilon_{\infty}}\right)^{1 / 4}
$$

While this is mathematically a length scale, it is not the quantity which is normally understood to be the Kolmogorov length based on an energy dissipation rate in (2.2) defined by $\varepsilon=v\left\langle\|D u\|_{2}^{2}\right\rangle L^{-3}$. How are we to make a comparison between $\lambda_{\mathrm{K}, \infty}$ and $\lambda_{\mathrm{K}}$ ? In so doing, one runs into an as yet insuperable problem. Using Gagliardo-Nirenberg inequalities to bound above an $L^{\infty}$ norm by a $W(N, p)$ norm (say), introduces the systern volume into the problem thereby sacrificing the intuitive notion of an intensive length scale determined only by local properties of the flow. Let us begin by writing down how many fixed (or bounded) scales exist against which we want to compare $\nu^{-1}\left\langle\|D u\|_{\infty}\right\rangle$.

- $\lambda_{0}^{-2}=L^{-2}+\lambda_{f}^{-2}$; the box length $L$ and the smallest scale in the forcing $\lambda_{f}$.

- $\lambda_{\mathrm{K}}=\left(\nu^{3} / \varepsilon\right)^{1 / 4}$; the Kolmogorov length.

- $\mu_{\mathrm{K}}=\left(\nu^{3} / \varepsilon_{f}\right)^{1 / 4}$; the equivalent of the Kolmogorov length for the forcing.

In their paper, Henshaw, Kreiss and Reyna [12] use the approximation

$$
\left\langle\|D u\|_{\infty}\right\rangle \approx L^{-3 / 2}\left\langle\|D u\|_{2}\right\rangle
$$

which makes

$$
\ell_{N, r}^{-2} \leqslant c_{N, r} \lambda_{\mathrm{K}, \infty}^{-2}+\lambda_{0}^{-2} \approx c_{N, r} \lambda_{\mathrm{K}}^{-2}+\lambda_{0}^{-2} .
$$

Physically, this approximation assumes that the dissipation in the flow is close to its spatial average, and that deviations away from this state do not contribute to the time average. This assumption can only be true for those periods in the flow in between intermittent bursts in the vorticity field. Thus we expect the conventional Kolmogorov length to resolve the flow during the quiescent periods only when (2.3) holds. The phenomenon of spatial intermittency, however, is associated with the energy dissipation being concentrated on a set of fractal dimension lower than the background space [20]. This leads to a fundamental violation of (2.3).

To see how far $\|D u\|_{\infty}$ is allowed to fluctuate away from $\|D u\|_{2}$ both in the time averaged sense and in the limsup sense, we prove 
Theorem 3. In $3 d$,

$$
\left\langle\|D u\|_{\infty}\right\rangle \leqslant c_{1} v^{-3}\left[\left\langle\|D u\|_{2}^{4}\right\rangle+\left\|D u_{f}\right\|_{2}^{4}\right]+c_{2} v \lambda_{0}^{-2}+L^{-3 / 2}\left\langle\|D u\|_{2}\right\rangle
$$

and

$\overline{\lim }_{t \rightarrow \infty}\|D u\|_{\infty} \leqslant c_{1} v^{-3}\left[\overline{\lim }_{t \rightarrow \infty}\|D u\|_{2}^{4}+\left\|D u_{f}\right\|_{2}^{4}\right]+c_{2} \nu \lambda_{0}^{-2}+\dot{L}^{-3 / 2} \overline{\lim }_{t \rightarrow \infty}\|D u\|_{2}$

There is also the following corollary which turns (2.5) and (2.6) into inequalities in the vorticity:

Corollary 1 .

$$
\left\langle\|\omega\|_{\infty}\right\rangle \leqslant c_{1} \nu^{-3}\left[\left\langle\|\omega\|_{2}^{4}\right\rangle+\left\|\omega_{f}\right\|_{2}^{4}\right]+L^{-3 / 2}\left\langle\|\omega\|_{2}\right\rangle+c_{2} \nu \lambda_{0}^{-2}
$$

and

$\varlimsup_{\lim }^{t \rightarrow \infty}\|\bar{\omega}\|_{\infty} \leqslant c_{1} v^{-3}\left[\overline{\lim }_{t \rightarrow \infty}\|\omega\|_{2}^{4}+\left\|\omega_{f}\right\|_{2}^{4}\right]+L^{-3 / 2} \overline{\lim }_{t \rightarrow \infty}\|\omega\|_{2}+c_{2} \nu \lambda_{0}^{-2}$.

Theorem 3, equation (2.5) demonstrates that

$\left\langle\kappa_{N, r}^{2}\right\rangle \leqslant c \lambda_{\mathrm{K}_{, \infty}}^{-2} \leqslant c(\underbrace{\lambda_{\mathrm{K}}^{-2}+\lambda_{0}^{-2}+\mu_{\mathrm{K}}^{-2}}_{\text {bounded terms }})+v^{-4}(\underbrace{\left\langle\|D u\|_{2}^{4}\right\rangle}_{\text {no control }}+\left\|D u_{f}\right\|_{2}^{4})$.

The first of the two extra terms with the $\nu^{-4}$ coefficient, despite the disadvantage of being $L$ dependent, is symbolic in pointing us to a mechanism of how large fluctuations to smaller scales than $\lambda_{\mathrm{K}}$ cannot be precluded. $\left\langle\|D u\|_{2}^{4}\right\rangle$ is, in effect, the part which might become singular $\dagger$ in finite time should regularity fail. But even if it does not, the $v^{-4}$ coefficient is significant in allowing this term to become large for high Reynolds numbers if the suprema in the time averages are reached. In consequence, we can see that $\lambda_{\mathrm{K}}$ is the natural scale only during those time periods between rare events when the vorticity in the fluid remains near its spatial average. These rare events will only occur if the fluid takes advantage of the slack allowed between the $L^{\infty}$ and $L^{2}$ norms in the theorem 3 . Can we say exactly how short the length scales are down to which these rare events go? By these methods alone, the answer to this is ' $N o$ '.

Theorem 3 is, in effect, only making a statement about what can happen as opposed to what will happen. Can we predict whether these vorticity bursts will actually occur? Nothing in our present methods indicate that they must happen nor do we have any handle on the possible sets of initial data that might cause them. Stepping into the realm of physics, it is not unreasonable to suppose that they are more likely to occur if the $3 d$ Euler equations exhibit finite time singularities. While this behaviour is not proven, it is generally believed that such singularities do occur (see the reviews by Majda $[2,3]$ ). A parallel can be drawn with the $2 d / 3 d$ complex Ginzburg Landau equation [21] where it is known rigorously that its inviscid limit, the Nonlinear Schrödinger (NLS) equation, exhibits finite time singularities.

Finally, as we have already compared $\|D u\|_{\infty}$ with $\|D u\|_{2}$, it is reasonable to ask whether any results of this type can be found for higher derivatives. Again, using the $\kappa_{N, r}$, the natural result is

† If the proof in theorem 3 is done slightly differently, we get $\left\langle\kappa_{N, r}^{2}\right\rangle \leqslant\left\langle\kappa_{N, r}^{3}\right\rangle^{1 / 2}\left\langle\|D u\|_{2}^{2}\right\rangle^{1 / 2}$. While $\left\langle\|D u\|_{2}^{2}\right\rangle$ is bounded above, this calculation illustrates the well known closure problem in turbulence where high moments control low moments. 
Theorem 4. For $p \geqslant 0, \omega_{f}=\operatorname{curl} u_{f}$,

$\left\langle\frac{\left\|\operatorname{curl}^{p} \omega\right\|_{\infty}}{\left\|\operatorname{curl} l^{p} \omega\right\|_{2}+\left\|\operatorname{curl} p^{p} \omega_{f}\right\|_{2}}\right\rangle \leqslant c_{1} \nu^{-3}\left[\left\langle\|\omega\|_{2}^{4}\right\rangle+\left\|\omega_{f}\right\|_{2}^{4}\right]+c_{2} \lambda_{0}^{-3 / 2}+L^{-3 / 2}$.

Apart from the constants, the right-hand side of equation (2.10) is independent of $p$.

\section{Properties of $\kappa_{N, r}$}

In section 1 it was demonstrated that $\left\langle\kappa_{N, r}^{2}\right\rangle$ form a set of squared inverse lengths with the $\kappa_{N, r}$ defined as

$$
\kappa_{N, r}=\left(\frac{F_{N}}{F_{r}}\right)^{1 / 2(N-r)}
$$

Here we take the argument further and show how they can be thought of as the natural mathematical objects through which some known Navier-Stokes results can be derived in a simple and elegant way. In fact we will shortly show how they also arise naturally in a result of Beale et al [1] concerning the Euler equation. In the language of the $H_{N}$ of section 1, Leray's inequality tells us that $\left\langle H_{1}\right\rangle$ is bounded above in any dimension. In the language of the $\kappa_{N, r}$, this translates to $\left\langle\kappa_{1,0}^{2}\right\rangle \leqslant \lambda_{0}^{-2}$ for both the $2 d$ and $3 d$ casest. The $\kappa_{N, r}$ are ordered in an obvious way

$$
\begin{array}{ll}
\kappa_{N_{1}, r}(t) \leqslant \kappa_{N_{2}, r}(t) & r<N_{1}<N_{2} \\
\kappa_{N, r_{1}}(t) \leqslant \kappa_{N, r_{2}}(t) & r_{1}<r_{2}<N .
\end{array}
$$

Studying the possibility of control over, or of singularity formation in, this string is therefore equivalent to studying the problem of regularity and/or its breakdown.

Theorem 5. For the $3 d$ Navier-Stokes equations with $r<N-1$

$$
(N-r) \dot{\kappa}_{N, r} \leqslant c_{N, r}\left(\|D u\|_{\infty}+\nu \lambda_{0}^{-2}\right)-\nu \kappa_{N, r}\left(\kappa_{N, r}^{2}-\kappa_{r+1, r}^{2}\right) .
$$

Since $\kappa_{r+1, r} \leqslant \kappa_{N, r}$ for $r+1<N$, the last set of terms in (3.4) are negative definite and can be removed. Then a simple integration with respect to time gives

$$
\kappa_{N, r}(t) \leqslant \kappa_{N, r}(0) \exp \left[\int_{0}^{t} c_{N, r}\left(\|D u\|_{\infty}(\tau)+\nu \lambda_{0}^{-2}\right)\right] \mathrm{d} \tau .
$$

This is, in effect, the equivalent of the Beale et al result [1] (which is implicit in their paper); namely, that if regularity breaks down at $t^{*}<\infty$, then $\int_{0}^{t^{*}}\|D u\|_{\infty}(s) \mathrm{d} s$ must blow up at $t^{*}$. No milder singularities are possible (see $[2,3])$. Since the last two terms in theorem 5 can be removed in the inequality, the only part dependent on $v$ and $f$ is the $\nu \lambda_{0}^{-2}$ term. For the Euler equations $\nu=0$ and $f=0$. This term vanishes from the theorem but the rest remains. Hence we have the equivalent result for the Euler equations. This reflects the joint results on the Euler and Navier-Stokes equations found by Constantin [22]. It is nice, however,

† We remind the reader that in $2 d$ alone it is also true that $\left\langle\kappa_{2,1}^{2}\right\} \leqslant \lambda_{0}^{-2}$, which is the generalization of $\left\langle H_{2}\right\rangle$ being bounded. 
that the dynamic wavenumber itself is the quantity which is shown to be controlled by the time integral of $\|D u\|_{\infty}$. The implications of this are discussed in section 4.

If, however, we want to convert estimates in $\|D u\|_{\infty}$ into ones in $\|\omega\|_{\infty}$, it is necessary to pay a price by introducing the system length $L$ explicitly. The key to this is the inequality of Beale et al [1] which shows how $\|D u\|_{\infty}$ is controlled by $\|\omega\|_{\infty}$ on the domain $[0,1]^{3}$ :

$$
\|D u\|_{\infty} \leqslant c\left[1+c\|\omega\|_{\infty}\left(1+\log ^{+} H_{3}\right)\right]+\|\omega\|_{2} .
$$

This inequality can be modified for the periodic domain $[0, L]^{3}$ into

\section{Lemma 1.}

$$
\|D u\|_{\infty} \leqslant c_{N, r}\|\omega\|_{\infty}\left[1+\log \left(L \kappa_{N, r}\right)\right]+L^{-3 / 2}\|\omega\|_{2}
$$

for $N \geqslant 3$ and $0 \leqslant r<N$. The + sign on the logarithm is defined such that $\log ^{+} a=\log a$ for $a \geqslant 1$ and $\log ^{+} a=0$ otherwise.

The main point is that the $\kappa_{N, r}(N \geqslant 3)$ are the natural 'mediators' $\dagger$ between $\|D u\|_{\infty}$ and $\|\omega\|_{\infty}$. Another consequence of the Beale et al result in the form given in lemma 1 is if we want to estimate the $\kappa_{N, r}$ explicitly as functions of time in terms of the time integral of $\|\omega\|_{\infty}$. An integration of the result of theorem 5 and lemma 1 gives

$$
\kappa_{N, r}(t) \leqslant c L^{-1} \exp \left(\int_{0}^{t} g\left(t^{\prime}\right) \exp \left[I(t)-I\left(t^{\prime}\right)\right] \mathrm{d} t^{\prime}\right)
$$

where

$$
I(t)=\int_{0}^{t}\|\omega\|_{\infty}(\tau) \mathrm{d} \tau
$$

and

$$
g(t)=v \lambda_{0}^{-2}+L^{-3 / 2}\|\omega\|_{2}
$$

The result for the Euler equations is recovered by putting $f=0$ and $v=0$. Here we have a solution of the differential inequality for both the Euler and the Navier-Stokes equations in terms of the $\kappa_{N, r}$, instead of the $H_{N}$ norms alone [1].

Finally, instead of considering how $\int_{0}^{\infty}\|D u\|_{\infty}(\tau) \mathrm{d} \tau$ controls the scales, it is possible to see how upper bounds on the $\kappa_{N, r}$ can become singular (we take the case $f=0$ ). Using the Gagliardo Nirenberg inequality for $N \geqslant 3$

$$
\|D u\|_{\infty} \leqslant c \kappa_{N, 0}^{5 / 2}\|u\|_{2}
$$

and using this in theorem 5 , we integrate with respect to time (ignoring the $\nu \lambda_{0}^{-2}$ term which does not change the nature of the result), we obtain

$$
\left[\kappa_{(N, 0)}(0)\right]^{-5 / 2}-\left[\kappa_{(N, 0)}(t)\right]^{-5 / 2} \leqslant c \int_{0}^{t}\|u\|_{2}(s) \mathrm{d} s
$$

† As an aside, this result, together with (3.7) and theorem 1, lead us to a curious result in which we are able to find an estimate in terms of $\left\langle\|\omega\|_{\infty}\right\rangle$, not for $\left\langle\kappa_{N, r}^{2}\right\rangle$ but for $\left\langle\kappa_{N, r}\right\rangle^{2}$. Specifically, in $3 d$ and for $N \geqslant 3$, $\left\langle\kappa_{N, r}\right\rangle^{2} \leqslant c Q\left[1+\log \left(L^{2} Q\right)\right]$ where $Q=\lambda_{\mathrm{k}}^{-2}+\lambda_{0}^{-2}+c \nu^{-1}\left\langle\|\omega\|_{\infty}\right\rangle$. The explicit system size dependence resides only in the logarithm. However, $\left\langle\kappa_{N, r}\right\rangle^{2}$ is less than the length scale defined in definition 2. 
which, from the Navier-Stokes equations, is itself bounded by

$$
G(t) \equiv \int_{0}^{t}\|u\|_{2}(s) \mathrm{d} s \leqslant c L^{-2} \nu^{-1}\|u\|_{2}(0)\left[1-\exp \left(-c L^{2} v t\right)\right]
$$

In combination with (3.12), this gives

$$
\kappa_{N, 0}(t) \leqslant c\left[\kappa_{N, 0}(0)^{-5 / 2}-G(t)\right]^{-2 / 5} .
$$

The solution has no singularities if

$$
\left[\kappa_{N, 0}(0)\right]^{-5 / 2}>c L^{-2} \nu^{-1}\|u\|_{2}(0)
$$

For $N \geqslant 3$, this result can be converted into

$$
\kappa_{N, 0}(0)<L^{-1}\left[c_{N} R_{e}\right]^{-2 / 5}
$$

where the Reynolds number $R_{e}$, defined in terms of the initial conditions is

$$
R_{e}=\frac{L^{-1 / 2}\|u\|_{2}(0)}{\nu}
$$

This is equivalent to the well known result of Ladyzhenskaya [15]; in this case, regularity can be achieved for high Reynolds numbers at the price of having the initial data on the third derivative very small.

\section{Conclusion}

\subsection{Levels of scales in $3 d$}

One of the main tools in this paper in examining length scales has been the construction of the dynamic 'wavenumbers' $\kappa_{N, r}(t)$ which, for $N \geqslant 3$, have the property $\left\langle\kappa_{N, r}^{2}\right\rangle \leqslant$ $c_{N, r} \nu^{-1}\left\langle\|D u\|_{\infty}\right\rangle+\lambda_{0}^{-2}$. How do these scales compare with those found via the attractor dimension, and the number of determining modes respectively? In $3 d$, they coincide with the $v^{-1}\left(\|D u\|_{\infty}\right)$ result of Constantin, Foias and Temam $[5,4]$ and Foias et al [7]. As we have pointed out in section 1 , they also coincide with those found in $[11,12]$. The fact that we have taken a time average could mean that some information about rare events in the flow may has been lost. We can conclude therefore that length scales may exist on three different levels.

- The first is the Kolmogorov length which will be the prevalent scale $\lambda_{\mathrm{K}}$ when $D u$ lies close to its spatial average: i.e. $\left\langle\|D u\|_{\infty}\right) \approx L^{-3 / 2}\left\langle\|D u\|_{2}\right)_{\text {: }}$ This scale will be sufficient to resolve the flow during those long intervals when it remains quiescent. Indeed, there must be long time intervals between large intermittent events so as not to violate averages.

- If an intermittent event occurs but the flow remains regular, then during this event, the minimum length scale could become considerably shorter than $\lambda_{\mathrm{K}}$, although down to what leyel it may go we cannot say without a regularity proof (see section 2).

- Even smaller scales than these are possible during events which our time average $\left\langle\kappa_{N, r}^{2}\right\rangle$ has averaged out, thereby missing some information. These are the smallest scales of all and are ones which, realistically, are given by the $\kappa_{N, r}$ themselves without the operation 
of the time average. We can write this inverse scale as (length) ${ }^{-1} \equiv \overline{\lim }_{t \rightarrow \infty} K_{N, r}$. As we have already found from theorem 5 ,

$$
(N-r) \kappa_{N, r}(t) \leqslant \kappa_{N, r}(0) \exp \int_{0}^{t}\left(c_{N, r}\|D u\|_{\infty}(\tau)+\nu \lambda_{0}^{-2}\right) \mathrm{d} \tau .
$$

This result returns us to the Beale et al result [1] for the $3 d$ Euler equations, where it is shown that $\int_{0}^{t}\|D u\|_{\infty}(\tau) \mathrm{d} \tau$ controls singularity formation. We cannot go beyond this without a regularity proof if the main part of the estimate is to be independent of system volume. If, however, we are willing to sacrifice intensivity then it is possible to find a limsup estimate directly for $\kappa_{N, 0}$ in terms of the $H_{1}$ norm.

Theorem 6. In $d$ dimensions and for $N>(4 / 4-d)$

$$
\varlimsup_{t \rightarrow \infty} \kappa_{N, 0}^{2} \leqslant c L^{-2} c_{N} Q^{\frac{2(N-1)}{(4-d) N-4}}
$$

where $Q=v^{-2} L^{4-d} \overline{\lim }_{\mathfrak{f} \rightarrow \infty} F_{1}$.

Since the $\kappa_{N, 0}$ are ordered such that $\kappa_{N_{2}, 0} \leqslant \kappa_{N_{2}, 0}$ for $N_{1}<N_{2}$, we must take the minimum of the right-hand side of the estimate in theorem 6 , namely $Q^{\frac{2}{(4-d)}+\varepsilon}$. For $d=3$, an examination of this shows that it is similar to the upper bound on the length scale derived in equation (2.9) using the time average. The $Q^{2}$ estimate, in effect, when all the factors of $L$ are accounted for, gives $v^{-4} \overline{\lim }_{t \rightarrow \infty} F_{1}^{2}$ : there we had $v^{-4}\left\langle F_{1}^{2}\right\rangle$. Although we would expect the latter to be higher, this is the only disparity between the two. Neither of these, (2.9) or (4.2), can be sharp because of the price paid by introducing the system volume into the problem and, with present methods, they are indeterminate. This inability to get a system size independent estimate is, we believe, due to the lack of a regularity proof. In the $2 d$ case, when the Navier-Stokes equations are known to be regular, we are on surer ground in making comparisons between $\left\langle\kappa_{N, 0}^{2}\right\rangle$ and $\left(\overline{\lim }_{t \rightarrow \infty} \kappa_{N, 0}\right)^{2}$.

\subsection{Length scales in the $2 d$ Navier-Stokes case}

While no explicit estimate for $\left\langle\kappa_{N, r}^{2}\right\rangle$ has been determined in $3 d$ this can be achieved very rapidly in the $2 d$ case:

Theorem 7. In $2 d$

$$
\left(\frac{\ell_{N, r}}{L}\right)^{-2} \equiv\left(L^{-1} \kappa_{N, r}\right)^{2} \leqslant c \mathcal{G}(1+\log \mathcal{G})^{1 / 2}
$$

where $\mathcal{G}=v^{-2} L^{2}\|f\|_{2}$ is the Grashof number.

In the proof in section 5 we have used the fact that $\left\langle F_{2}\right\rangle \leqslant v^{2} L^{-4} \mathcal{G}^{2}$. We have also ignored the $\lambda_{0}^{-2}$ terms. The estimate in (4.3) coincides with the number of determining modes given in $[7,8]$ but it is larger than the equivalent scale given by the attractor dimension $[13,5,6,23]$

$$
\left(\frac{\ell}{L}\right)^{-2} \leqslant c \mathcal{G}^{2 / 3}(1+\log \mathcal{G})^{1 / 3}
$$

The left-hand sides of (4.3) and (4.4) are the number of degrees of freedom in a $2 d$ system $\mathcal{N}=(L / l)^{2}$ which represents the number of 'eddies' of typical size $\ell^{2}$ that can fit in the 
system volume $L^{2}$. The estimate in (4.3) for our time averaged inverse scale is smaller than that found for the length scale based on the attractor dimension. This discrepancy is not due to a lack of sharpness in our inequalities but reflects the fact that we are comparing different measures of the dynamics. The way the attractor dimension is computed depends only on $\left\langle F_{2}\right\rangle$ and on no norms higher than this. During intermittent fluctuations, it is possible that the magnitude of norms higher than $F_{2}$ may be considerable, if only for a short time interval. The estimate in (4.3) picks up more information because it takes into account every derivative through the use of the ladder theorem. This indicates that a length scale based on the attractor dimension is measuring grosser features in the flow $\dagger$, whereas $\left(\kappa_{N, r}^{2}\right)$, together with the scales found by the number of determining modes, is sensitive to fluctuations down to smaller scales. In $2 d$, it is possible to compute an estimate for $\kappa_{N, r}(t)$. This can be found from theorem 6 for $\kappa_{N, 0}$. This can also be computed for higher values of $r>0$. In terms of $\mathcal{N}$, this is given by

$$
\mathcal{N} \equiv\left(L \overline{\lim }_{t \rightarrow \infty} \kappa_{N, r}\right)^{2} \leqslant c_{N} \mathcal{G}^{2} .
$$

For $r>0$ this is easily found by estimating $F_{N}$ from the ladder in terms of $F_{1}$ (which in $2 d$ is bounded) and then using the fixed lower bound on $F_{r}$. We leave this calculation to the reader.

In summary, estimates in $2 d$ for $\mathcal{N}=(L / \ell)^{2}$ vary from

- The attractor dimension: $\mathcal{N}_{1} \leqslant c \mathcal{G}^{2 / 3}(1+\log \mathcal{G})^{1 / 3}$.

- The time average definition (and the number of determining modes) which gives the estimate: $\mathcal{N}_{2} \leqslant c \mathcal{G}(1+\log \mathcal{G})^{1 / 2}$

- The scale calculated via the limsup of $\kappa_{N, r}: \mathcal{N}_{3} \leqslant c \mathcal{G}^{2}$.

To investigate a further example of what the time averages may miss in the estimates, we also briefly mention another problem where we have both regularity and the ability to make comparisons between estimates where there are different types of dynamics. This example is the complex Ginzburg Landau (CGL) equation [21].

\subsection{The example of the CGL equation}

The complex Ginzburg Landau equation on a periodic domain $\Omega \equiv[0,1]^{2}$,

$$
A_{t}=R A+(1+\mathrm{i} \nu) \Delta A-(1+\mathrm{i} \mu) A|A|^{2}
$$

has various regions in the $(\mu-\nu)$ plane where estimates are qualitatively different (see [21] for details). We consider $R>0$ to be the control parameter representing the driving in the PDE. The parameters $\mu, \nu$ are of either sign and are specific to the application. The ladder theorem in this case is

$$
\frac{1}{2} \dot{F}_{N} \leqslant-b_{N} \frac{F_{N}^{1+1 / s}}{F_{N-s}^{1 / s}}+\left(c\|A\|_{\infty}^{2}+N R\right) F_{N}
$$

where

$$
F_{N}=\int_{\Omega}\left[\left|D^{N-1} A\right|^{2}+\alpha_{N}|A|^{2 N}\right] \mathrm{d} x+R^{N}
$$

where the $\alpha_{N}$ are a set of strictly positive constants and $b_{N}>0$ is related to them. There is an equivalent set of $\kappa_{N}, r$, defined in exactly the same combination of the $F_{N}$ as in section 1 
for the Navier-Stokes equations. We will not go through every point in the analysis here; instead we describe the steps. Firstly, we find an estimate for $\|A\|_{\infty}$ in terms of $\|D A\|_{\infty}$ and $\|A\|_{2}$ and then use the same procedure as in the proof $\dagger$ of theorems 2,3 and 4 . Then we find that

$$
\left\langle\kappa_{N, 1}^{2}\right\rangle \leqslant c(\nu, \mu) R^{3}+R .
$$

The point to note about this equation is that it is valid over the whole $(\mu, \nu)$ plane and the parameter dependence on $\mu$ and $\nu$ is only in the multiplicative constant. While it is possible to improve this estimate to $R$ in the so-called 'weak' region $(|\mu|,|\nu|$ small) described in [21], where all solutions are strapped down close to their averages, the estimate given in (4.9) is valid in the so-called 'strong' region where $|\mu|,|\nu|$ are large. It is in this region where one is close to the finite time singularity in the NLS equation. Moreover, the discrepancy between $L^{\infty}$ and $L^{2}$ norms can be vast if both $R$ and $|\nu|$ are chosen to be large. This is reflected in the estimate

$$
\overline{\lim }_{t \rightarrow \infty}\|A\|_{\infty}^{2} \leqslant R+c R^{|v|+1} .
$$

Altogether, therefore, we expect spatially and temporally local intermittent events which can possibly $\ddagger$ get larger in amplitude because the exponent on the right-hand side of (4.10) is increasing with $|\nu|$. It is clear, however, that the scale computed via the time average in (4.9) is not completely sensitive to the increasing magnitude of these fluctuations (except in the constant $c(\mu, \nu)$ ). To pick up the 'spikes', one would have to use the other definition given above for the Navier-Stokes equations: namely, $\overline{\lim }_{t \rightarrow \infty} \kappa_{N, r}(t)$. A repetition of the calculation equivalent to theorem 7 gives

$$
L^{2}\left(\overline{\lim }_{t \rightarrow \infty} \kappa_{N, 1}\right)^{2} \leqslant R+c_{N} R^{|\nu|+1}
$$

\section{Theorem proofs}

In previous sections we promised to provide the proofs of the various theorems stated in this paper.

Proof of theorem 1 . With the definition

$$
H_{N}=\sum_{i=1}^{d} \sum_{|n|=N} \int\left|D^{n} u_{i}\right|^{2} \mathrm{~d} x \equiv\left\|D^{N} u\right\|_{2}^{2}
$$

we know from [10] that the $H_{N}$ satisfy

$$
\frac{1}{2} \dot{H}_{N} \leqslant-\nu H_{N+1}+c H_{N}\|D u\|_{\infty}+H_{N}^{1 / 2}\left\|D^{N} f\right\|_{2} \text {. }
$$

Then it can be demonstrated, on a periodic domain [24] for $q<M$, that

$$
H_{M}^{p+q} \leqslant H_{M+p}^{q} H_{M-q}^{p}
$$

I In parallel with the minor restriction on the forcing in theorem 2, here we need $R>1$. For $0<R<1$, the attractor is the origin.

$\ddagger$ We say 'possibly' because, to date, no definitive numerical study of the spiky region has been performed. 
whence, with $M=N, p=1$ and $q=s$

$$
\frac{1}{2} \dot{H}_{N} \leqslant-v \frac{H_{N}^{1+1 / s}}{H_{N-s}^{1 / s}}+c H_{N}\|D u\|_{\infty}+H_{N}^{1 / 2}\left\|D^{N} f\right\|_{2} .
$$

The only difference in the statement of the result given in (5.4) from the way it is expressed in [10] is that the first term on the right-hand side allows a drop of $s$ rungs in the ladder instead of one rung. In section 1 and section 2 we have examined time averages of ratios of norms and, without taking proper account of the forcing, the last term in (5.4) potentially creates problems because it is possible that $H_{N}$ may get very close to zero, thereby causing this term to become disproportionately large. It is therefore necessary to put together all the components of the forcing along with the components of the $H_{N}$ to make one variable, as in (1.4).

Since $u_{f}$ is independent of time there is no contribution to the left-hand side. To (5.2) we add and subtract $v\left\|D^{N+1} u_{f}\right\|_{2}^{2}$ and then apply the inequality (5.3) to the negative part only to bring it into the same form as its equivalent negative definite term in (5.4). Then an application of the Hölder-Schwartz inequality produces the negative definite term on the right-hand side of (1.5). The $\tau_{0}^{-1} F_{N}$ term arises from the last term in (5.2) while $\nu \lambda_{f}^{-2}$ arises from the $v\left\|D^{N+1} u_{f}\right\|_{2}^{2}$ term in the following way:

$$
\nu\left\|D^{N+1} u_{f}\right\|_{2}^{2} \leqslant v \frac{\left\|D^{N+1} u_{f}\right\|_{2}^{2}}{\left\|D^{N} u_{f}\right\|_{2}^{2}} F_{N} \leqslant v \lambda_{f}^{-2} F_{N}
$$

where $\lambda_{f}$ is the cut-off scale.

Proof of theorem 2. The proof is based on dividing the $F_{N}$ ladder in theorem 1 by $F_{N}$ and averaging over time. The only matter it is necessary to mention is the lower bound on $F_{N}$. We need to calculate a lower bound on

$$
\left\langle\frac{\dot{F}_{N}}{F_{N}}\right\rangle=\sup _{F_{N}(0)} \lim _{t \rightarrow \infty} \frac{1}{t}\left[\log F_{N}(t)-\log F_{N}(0)\right] .
$$

The second term is zero as the argument is constant. The first term is bounded below by zero provided $F_{N}>1$. This therefore places a minor restriction on the forcing. The two cases $(1,0)$ and $(2,1)$ can be worked out from the individual ladders for $F_{0}$ and $F_{1}$ where one divides through by $F_{0}$ and $F_{1}$ respectively.

\section{Proof of theorem 3.}

Step 1: proof of (2.5) and (2.7). The key to the proof is the dynamic wavenumbers $\kappa_{N, r}$ which we can use after interpolating between $L^{\infty} \& L^{2}$ of $D u$ with $N \geqslant 3$

$$
\|D u\|_{\infty} \leqslant c\left\|D^{N} u\right\|_{2}^{a}\|D u\|_{2}^{1-a}+L^{-3 / 2}\|D u\|_{2} \leqslant c F_{N}^{a / 2} F_{1}^{(1-a) / 2}+L^{-3 / 2}\|D u\|_{2}
$$

which, in turn

$$
\leqslant c \kappa_{N, 1}^{3 / 2} F_{1}^{1 / 2}+L^{-3 / 2}\|D u\|_{2}
$$

Note that the wavenumber $\kappa_{N, 1}$ appears in the correct dimensional way. The time average of (5.8) obeys

$$
\left\langle\|D u\|_{\infty}\right\rangle \leqslant c\left\langle\kappa_{N, 1}^{2}\right\rangle^{3 / 4}\left\langle F_{1}^{2}\right\rangle^{1 / 4}+L^{-3 / 2}\left\langle\|D u\|_{2}\right\rangle
$$


Now we can use theorem 1 with $r=1$ (i.e. $s=N-1$ ) to obtain

$$
\left\langle\|D u\|_{\infty}\right\rangle \leqslant\left[c \nu^{-1}\left\langle\|D u\|_{\infty}\right\rangle+\lambda_{0}^{-2}\right]^{3 / 4}\left\langle F_{1}^{2}\right\rangle^{1 / 4}+L^{-3 / 2}\left\langle\|D u\|_{2}\right\rangle .
$$

Using Young's inequality on the right-hand side of (5.10), we combine the terms in $\|D u\|_{\infty}$ and finally obtain

$$
\left\langle\|D u\|_{\infty}\right\rangle \leqslant c_{1} \nu^{-3}\left\langle\|D u\|_{2}^{4}\right\rangle+c_{2} \nu^{-3}\left\|D u_{f}\right\|_{2}^{4}+c_{3} \nu \lambda_{0}^{-2}+L^{-3 / 2}\left\langle\|D u\|_{2}\right\rangle
$$

which is (2.5). The corollary to this, (2.7), follows since $\|\omega\|_{\infty} \leqslant\|D u\|_{\infty}$ and $\|\omega\|_{2}=$ $\|D u\|_{2}$.

Step 2: Proof of (2.6) and (2.8). Using interpolation with $b=3 / 2 \mathrm{~N}$

$\|D u\|_{\infty} \leqslant c H_{N+1}^{b / 2} H_{1}^{(1-b) / 2}+L^{-3 / 2}\|D u\|_{2} \leqslant c F_{N+\mathrm{t}}^{b / 2} F_{1}^{(1-b) / 2}+L^{-3 / 2}\|D u\|_{2}$.

From theorem 1, let $N \rightarrow N+1 \& s=N \&$ solve the ladder (1.5) to get

$$
\varlimsup_{t \rightarrow \infty} F_{N+1} \leqslant\left[c v^{-1} \varlimsup_{\lim _{t \rightarrow \infty}}\|D u\|_{\infty}+\lambda_{0}^{-2}\right]^{N} \overline{\lim }_{t \rightarrow \infty} F_{1}+L^{-3 / 2} \overline{\lim }_{t \rightarrow \infty}\|D u\|_{2}
$$

whence (5.12) becomes

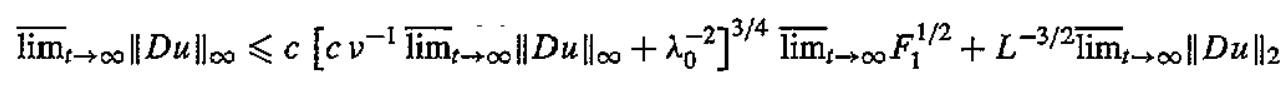

and so, after Young's inequality,

$\overline{\lim }_{t \rightarrow \infty}\|D u\|_{\infty} \leqslant c_{1} v^{-3} \overline{\lim }_{t \rightarrow \infty}\|D u\|_{2}^{4}+c_{2} v^{-3}\left\|D u_{f}\right\|_{2}^{4}+c_{3} \nu \lambda_{0}^{-2}+L^{-3 / 2} \overline{\lim }_{t \rightarrow \infty}\|D u\|_{2}$.

The corollary to (2.8) is true for the same reasons given in step 1 .

Proof of theorem 4. To prove (2.10) we interpolate $D^{r} u$ between $L^{\infty}$ and $L^{2}$ going up to $N-r$ derivatives (with $N \geqslant r+2$ ). Then we find after division that

$$
\begin{aligned}
\left\langle\frac{\left\|D^{r} u\right\|_{\infty}}{\left\|D^{r} u\right\|_{2}+\left\|D^{r} u_{f}\right\|_{2}}\right\rangle \leqslant c\left\langle\kappa_{N, r}^{2}\right\rangle^{3 / 4}+L^{-3 / 2} \\
\quad \leqslant\left[c_{1} \nu^{-1}\left\langle\|D u\|_{\infty}\right\rangle+\lambda_{0}^{-2}\right]^{3 / 4}+L^{-3 / 2} \\
\leqslant c_{1} \nu^{-3}\left\langle\|D u\|_{2}^{4}\right\rangle^{3 / 4}+c_{2} v^{-3}\left\langle\left\|D u_{f}\right\|_{2}^{4}\right\rangle^{3 / 4}+c_{3} \lambda_{0}^{-3 / 2}+L^{-3 / 2}
\end{aligned}
$$

from (2.5). The result, with $r-1=p$, follows. 
Proof of lemma 1. Let us return to the proof in [1] in which those authors consider solutions for the velocity field $u(x, t)$ in three parts $u^{(1)}, u^{(2)}$ and $u^{(3)}$ through the Biot-Savart law. In what follows we shall take periodic boundary conditions on the domain $[0, L]^{3}$ which means that $u^{(3)}$ does not contribute. We shall follow their method of using a cut-off function with a radius $\rho$ for which $0 \leqslant \rho \leqslant L$ which is now in a finite volume and which is to be determined later. We pick up their proof only where we take a different route to theirs: the interested reader must turn to [1] for the full method. Following their proof, one can write

$$
\left\|D u^{(1)}\right\|_{\infty} \leqslant\|K\|_{p}\|D \omega\|_{q} \leqslant c \rho^{1-3 / q}\|D \omega\|_{q} .
$$

In [1] they took $p=4 / 3$ and $q=4$. Here we leave them free except they are under the restriction $q \geqslant 3$ with $1 / p+1 / q=1$, generalizing the proof in [1]. Now we use a Gagliardo Nirenberg instead of a Sobolev inequality:

$$
\|D \omega\|_{q} \leqslant c\left\|D^{N-1} \omega\right\|_{2}^{a}\|\omega\|_{2}^{1-a}
$$

where, with $N \geqslant 3$,

$$
a=\frac{3}{N-1}\left(\frac{5}{6}-\frac{1}{q}\right) \text {. }
$$

Consequently, the equivalent of equation (17) in [1] is

$$
\left\|D u^{(1)}\right\|_{\infty}^{2} \leqslant c \rho^{2(1-3 / q)} \kappa_{N, 1}^{2(N-1) a} F_{1}
$$

which finally gives, in their equation (21),

$$
\|D u\|_{\infty} \leqslant c \rho^{1-3 / q} \kappa_{N, 1}^{5 / 2-3 / q} F_{1}^{1 / 2}+c\|\omega\|_{\infty}\left[1-\log ^{+}\left(\rho L^{-1}\right)\right] .
$$

Now we choose

$$
\rho^{-1}=c \kappa_{N, 1}^{(5 q-6) / 2(q-3)} L^{3 q / 2(q-3)}
$$

which gives the result of the lemma.

Proof of theorem 5. We know that $\kappa_{N, r}^{2(N-r)}=F_{N} / F_{r}$ and so

$$
2(N-r) \dot{K}_{N, r} \kappa_{N, r}^{2(N-r)-1}=\frac{\dot{F}_{N}}{F_{r}}-\left(\frac{F_{N}}{F_{r}}\right)\left(\frac{\dot{F}_{r}}{F_{r}}\right) .
$$

It is now necessary to use two versions of the ladder. There is the version in theorem I where $s=N-r$. It is also necessary to have the reverse version where we need to go back a few steps in the proof to get

$$
\frac{1}{2} \dot{F}_{r} \geqslant-\nu F_{r+1}-\left(c\|D u\|_{\infty}+\nu \lambda_{0}^{-2}\right) F_{r}
$$

from which we conclude that

$$
(N-r) \dot{\kappa}_{N, r} \leqslant c_{N, r} \kappa_{N, r}\left(\|D u\|_{\infty}+\nu \lambda_{0}^{-2}\right)+\nu \kappa_{N, r}\left[\kappa_{r+1, r}^{2}-\kappa_{N, r}^{2}\right] .
$$

To show that the final bracket is negative or zero, we simply need to appeal to the fact that $\kappa_{r+1, r} \leqslant \kappa_{N, r}$ provided $r+1<N$. 
Proof of theorem 6 . We pursue a similar line to theorem 5 except that we take $r=0$. The positive term from the Laplacian in (3.4), $\kappa_{N, 0} \kappa_{1,0}^{2}=\kappa_{N, 0} F_{1} / F_{0}$. We obtain

$$
N \kappa_{N, 0} \leqslant-v \kappa_{N, 0}^{3}+\left(c_{N}\|D u\|_{\infty}+2 v \lambda_{0}^{-2}\right)+\nu \kappa_{N, 0}\left(F_{1} / F_{0}\right)
$$

Because of the way $F_{0}$ is defined we know that it has a lower bound which can be used in the last term. This lower bound is $F_{0} \geqslant \tau^{2}\|f\|_{2}^{2}$. From interpolation, we also know that

$$
\|D u\|_{\infty} \leqslant c \kappa_{N, 1}^{d / 2} F_{1}^{1 / 2} \leqslant c L^{d / 2(N-1)} \kappa_{N, 0}^{N d / 2(N-1)} F_{1}^{1 / 2}
$$

where we have used the fact that $F_{0} \leqslant L^{2} F_{1}$. Using this in equation (5.27), the $\|D u\|_{\infty}$ term in (5.28) is the strongest term competing with the $-\kappa_{N, 0}^{3}$ term and so we end up with an absorbing ball for $\kappa_{N, 0}$ which gives the result.

Proof of theorem' 7. We use the logarithmic inequality of Brezis and Gallouet which estimates a $2 d L^{\infty}$ norm in terms of $H_{1}$ but with a logarithmic correction which takes into account the $\mathrm{H}_{2}$ norm [25] (see also [23] for an easier and more informal proof). Taking into account our forcing terms, $\|D u\|_{\infty}$ can be written

$$
\|D u\|_{\infty} \leqslant c F_{2}^{1 / 2}\left[1+\frac{1}{2} \log \left(L^{2} \frac{F_{3}}{F_{2}}\right)\right] \text {. }
$$

Now we notice that $F_{3} / F_{2}=\kappa_{3,2} \leqslant \kappa_{N, r}$ for $2<r<N$ with $N>3$. Applications of first Cauchy's and then Jensen's inequality gives

$$
\left\langle\|D u\|_{\infty}\right\rangle \leqslant c\left\langle F_{2}\right\rangle^{1 / 2}\left[1+\frac{1}{2} \log \left(L^{2}\left(\kappa_{3,2}^{2}\right\rangle\right)\right]^{1 / 2} .
$$

Now we can appeal to theorem 1 to estimate $\left\langle\|D u\|_{\infty}\right\rangle$ by using the fact that $\left\langle F_{2}\right\rangle \leqslant$ $v^{2} L^{-4} \mathcal{G}^{2}$. In so doing we ignore the $\lambda_{0}^{-2}$ termis. To do this we take the logarithm of both sides and use the fact that $\log (1+\log Q) \leqslant \log Q$ for $Q \geqslant 1$. This gives the result.

\section{Acknowledgments}

We acknowledge helpful discussions with Peter Constantin, Ciprian Foias, Darryl Holm, David Levermore, Andy Majda and Derek Moore. MVB would like to thank the UKK SERC for a research assistantship and the CNR (Italy). CRD is supported on NSF Grants PHY8907755, PHY-8958506 and PHY 9214715. SJAM would also like to thank the UK SERC for a fees only studentship and the University of London for the Keddey Fletcher-Warr studentship.

\section{References}

[1] Beale J T and Kato T and Majda A 1984 Remarks on the breakdown of smooth solutions for the 3D Euler equations Commun. Math. Phys. 94 61-6

[2] Majda A 1986 Vorticity and the mathematical theory of incompressible fluid flow Commun. Pure Appl. Math. 39 187-220

[3] Majda A 1991 Vorticity, turbulence and acoustics in fluid flow SIAM Rev. 33 349-88

[4] Constantin P, Foias C and Temam R 1985 Attractors representing turbulent flows Memoirs of AMS (Providence, RI: American Mathematical Society)

[5] Constantin P and Foias C 1989 Navier-Stokes Equations (Chicago, IL: Chicago University Press) 
[6] Temam R 1988 Infinite dimensional dynamical systems in mechanics and physics Springer Applied Mathematics Series vol 68 (Berlin: Springer)

[7] Foias C, Manley O P, Temam R and Treve Y M 1983 Asymptotic analysis of the Navier-Stokes equations Physica D 9 157-88

[8] Constantin P, Foias C, Manley O P and Temam R 1985 Determining modes and fractal dimension of turbulent flows $J$. Fluid Mech. $150427-440$

[9] Manley O P and Treve Y M 1981 Minimum number of modes in approximate solutions to equations of hydrodynamics Phys. Lett. 82A 88-90

[10] Bartuccelli M, Doering C and Gibbon J D 1991 Ladder theorems for the 2D and 3D Navier-Stokes equations on a finite periodic domain Nonlinearity $4531-42$

[11] Henshaw W D, Kreiss $H O$ and Reyna $L G 1989$ On smallest scale estimates for the incompressible Navier-Stokes equations Theor. Comput. Fluid Dyn. 1 65-95

[12] Henshaw W D, Kreiss H O and Reyna L G 1991 Smallest scale estimates for the incompressible NavierStokes equations Preprint

[13] Constantin P, Foias C and Temam R 1988 On the dimension of the attractors in two-dimensional turbulence Physica D 30 284-96

[14] Foias C and Prodi $\mathrm{G} 1967$ Sur le comportement global des solutions non-stationnaires des equations de Navier-Stokes en dimension 2 Rend. Sem Mat. Padova 39 1-39

[15] Ladyzhenskaya O A 1969 The Mathematical Theory of Viscous Incompressible Flow 2nd edn (New York: Gordon and Breach)

[16] Serrin J 1963 The initial value problem for the Navier-Stokes equations Nonlinear Problems ed R E Langer (Madison, WI: University of Wisconsin Press)

[17] Temam R 1979 Navier-Stokes Equations, Theory and Numerical Analysis (Amsterdam: North-Folland)

[18] Temam R 1983 The Navier-Stokes equations and non-linear functional analysis CBMS-NSF Regional Conf. Series in Applied Mathematics (Philadelphia, PA: SIAM)

[19] Doering C R and Constantin P 1992 Encrgy dissipation in shear driven turoulence Phys, Rev, Lett. 69 1648-51

[20] Prasad R K, Meneveau C and Sreenivasan K R 1988 Multifractal nature of the dissipation field of passive scalars in fully developed turbulent flows Phys. Rev. Lett. 61 74-7

[21] Bartuccelli M, Constantin P, Doering C R, Gibbon J D and Gisselfält M 1990 On the possibility of soft and hard turbulence in the complex Ginzburg-Landau equation Physica D 44 421-44

[22] Constantin P 1986 Note on the loss of regularity for solutions of 3D incompressible Euler and related equations Commun. Math. Phys. 10431 I-29

[23] Doering C R and Gibbon J D 1991 Note on the Constantin-Foias-Temam attractor dimension for 2dimensional turbulence Physica D 48 471-80

[24] Bartuccelli M, Doering C R, Gibbon J D and Malham S J 1993 Lattice methods and the pressure field for solutions of the Navier-Stokes equations Nonlinearity $679-91$

[25] Brezis H and Gallouet T 1980 Nonlinear Schrödinger evolution equations Nonlinear Analysis, Theory, Methods and Applications 4 677-681 